\title{
Bayesian Inference Based MPPT for Dynamic Irradiance Conditions
}

\author{
Buvana Lefevre $^{\mathrm{a}, \mathrm{b}, *}$, Bert Herteleer ${ }^{\mathrm{a}, \mathrm{b}}$, Sven De Breucker ${ }^{\mathrm{b}, \mathrm{c}}$, Johan Driesen ${ }^{\mathrm{a}, \mathrm{b}}$ \\ ${ }^{a}$ KU Leuven, ESAT; imec; B-3001 Leuven, Belgium \\ ${ }^{b}$ Energyville, Thor Park 8300, B-3600 Genk, Belgium \\ ${ }^{c}$ VITO, Belguim
}

\begin{abstract}
We introduce a Bayesian inference based maximum power point tracker (BI-MPPT) and demonstrate it under static and dynamic irradiance conditions. BI-MPPT is based on a probability inference technique which uses the model of the photovoltaic (PV) module and accounts for noise in the system. Owing to the model-based approach, the tracker converges fast to the maximum power point of the PV module and is capable of tracking dynamically varying irradiance. We compare the proposed BI-MPPT to an optimized model-based P\&O tracker and show that the proposed tracker consistently outperforms the latter. In experiments conducted with an in-house built solar emulator, at low illumination, the BI-MPPT achieves a static efficiency of $99.9 \%$ and a dynamic efficiency of at the least $97.4 \%$ and outperforms the optimized P\&O tracker by about 10 percent point. When BI-MPPT is applied to I-V curve measurements of an outdoor PV installation, at moderate and high irradiances, the dynamic efficiency is between 98.92 and $99.61 \%$ yielding a 3 to 4 percent point improvement over the optimized $\mathrm{P} \& \mathrm{O}$.

Keywords: Maximum power point tracking, dynamic efficiency, perturb and observe, Bayesian inference
\end{abstract}

\section{Introduction}

Photovoltaic (PV) modules are prone to varying operating conditions due to diurnal and seasonal changes in the environment, changes in cloud cover and the immediate environment of the module. With a change in the operating conditions, the maximum power producible by a PV module also

\footnotetext{
${ }^{*}$ Corresponding author

Email address: buvana.lefevre@esat.kuleuven.be (Buvana Lefevre)
} 
varies. This maximum producible power is located at the maximum power point (MPP) of the output characteristics of the PV module. MPP tracking ensures the maximum transfer of power from the module to the load. Tracking the MPP of a PV module would be straightforward if accurately sensing the irradiance were less cumbersome or costly [1, 2]. The MPP can also be determined by performing regression and tracing out a part of or an entire $\mathrm{I}-\mathrm{V}$ curve, yet the noise in the system limits the speed of the successive measurements. Moreover, if the successive measurements are not fast enough, the irradiance and temperature may vary between samples, making this solution infeasible as well. Tracing the $\mathrm{I}-\mathrm{V}$ curve is best used in combination with other tracking techniques where it serves to reduce the error between the operating point and the MPP [3]. An ideal solution would instead be to employ a single tracking algorithm requiring as few measurements as possible from voltage and current sensors and accurately determining the operating point under dynamic irradiance conditions. However, these voltage and current measurements are often noisy and could introduce errors in determining the maximum power point [4, 5].

Of the maximum power point trackers (MPPTs) found in the literature [6 [14, the most popular are the hill climbing techniques and in particular perturb and observe $(\mathrm{P} \& \mathrm{O})$ based algorithms and incremental conductance $(\mathrm{InC})$ algorithms. The P\&O based techniques are, however, limited by their inherently oscillatory character and the presence of noise in the system. InC algorithm, by design, should overcome the oscillatory nature of $\mathrm{P} \& \mathrm{O}$, which is however difficult in practice due to noise in the system [5]. Both $\mathrm{P} \& \mathrm{O}$ and $\mathrm{InC}$ are inaccurate in tracking rapid irradiance changes [15]. To overcome these limitations and to improve the tracking accuracy, these algorithms are often optimized for a given PV system or use hybrid techniques. InC based algorithms that are improved in performance such as those that use power increment techniques are more complex in comparison which also leads to slower sampling than by $\mathrm{P} \& \mathrm{O}$ [16, 17. However, optimization of $\mathrm{P} \& \mathrm{O}$ to reduce oscillations and improve convergence increases its performance [18 21] to match that of a similarly optimized InC algorithm [5, 16, 22. Since the optimization is very specific to the system, any changes to the system will therefore require re-optimization of the relevant parameters.

Soft computing techniques are generally considered versatile in that they can work efficiently in noisy conditions and under dynamic irradiation. For example, neural networks based trackers are fast in converging to MPP but their accuracy relies on the extensive training that is required prior to operation. The network is often trained for the PV model parameters of a specific PV system 23. Thus, a change in the system would require re-training the neural network for those parameters. 
Particle swarm optimization is a method wherein the operating point is computed based on a comparison of the present and past values of several particles, thereby requiring multiple iterations per particle along with a database to store previous iteration values for each of them [24]. Non-linear prediction treats the $\mathrm{P}-\mathrm{V}$ curve as either a quadratic or a parabola and therefore requires multiple iterations to compute for a good fit for the parameters [25. Kalman filter is a tool that is good at estimating the state of the system from noisy measurements [26]. As a MPPT, however, Kalman filter is applied to a gradient descent process making the tracker prone to oscillations similar to that observed with $\mathrm{P} \& \mathrm{O}[27-29]$. Often computational intelligence based trackers work in coordination with P\&O. However, even with the additional complexity of the intelligent algorithms, the inherent properties of $\mathrm{P} \& \mathrm{O}$, such as oscillations, still persist in these hybrid approaches. For a detailed summary of soft computing techniques we refer the reader to the articles [30, 31].

We propose a Bayesian inference based maximum power point tracker (BI-MPPT) to determine the MPP of a PV module within a single iteration, using one pair of voltage and current measurement values. By probabilistic representation of this pair of voltage and current measurement values, we accommodate for noisy sensors. The single pair of voltage and current measurements serve as input to a simple network model whose output is an estimation of the irradiance value for the next iteration. In our experiments, we use a simple one-diode model for the PV module. In section 2, we define the MPPT problem and describe a state-of-the-art, optimized P\&O algorithm in section 2.1. In section 3 we introduce the proposed BI-MPPT algorithm. In section 4 we compare the proposed BI-MPPT and the optimized P\&O algorithm experimentally with an in-house built hardware-in-loop solar emulator. Using this experimental setup, we determine the algorithm's convergence speed and operation at static illumination conditions and demonstrate the tracking accuracy under dynamic conditions at low illumination. Furthermore, by subjecting the trackers to identical operating conditions using measurement data of medium and high irradiances in section 4.4. we validate the dynamic tracking accuracy of the proposed BI-MPPT algorithm. We give our conclusions in section 5

\section{Maximum power point tracking}

Tracing successive I-V characteristics of a PV module gives an evolution of MPP over time. We illustrate the evolution of MPP from P-V curve measurement data in figure 1. The curves were 


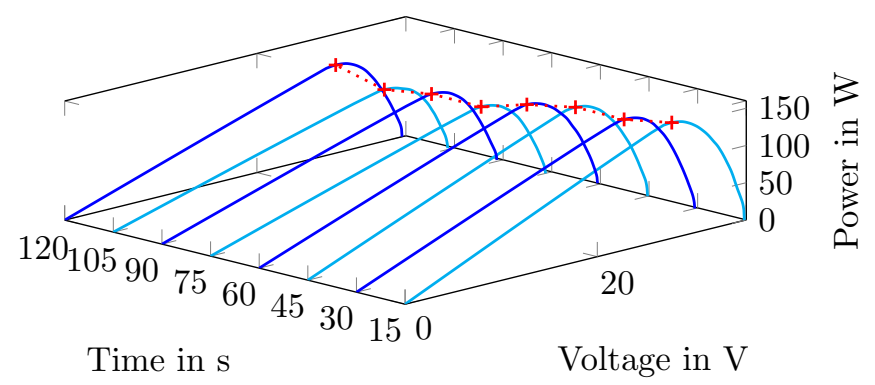

Figure 1: The consecutive P-V curves (- and - - -) measured every $15 \mathrm{~s}$ and their corresponding unique MPPs $(.+$.$) illustrate the variation of the maximum power point over time.$

traced out every $15 \mathrm{~s}$. The illustration shows that with a change in irradiance, $g$, the power at the MPP is not constant, as is the corresponding voltage.

The ideal MPPT is able to determine an operating point that corresponds to the desired MPP and is able to follow its evolution. The closer the operating point is to the MPP, the higher is the energy transferred from the PV module to the system. Thus, the accuracy in determining the MPP gives a measure of the performance of a tracker and is referred to as the efficiency of the tracker. The efficiency of a maximum power point tracker as given by the standard EN50530 : 2010 [32] is reproduced below.

$$
\eta_{m p p}=\frac{\sum_{j} i_{\text {meas }, j} v_{\text {meas }, j} \Delta t_{j}}{\sum_{k} i_{\mathrm{MPP}, k} v_{\mathrm{MPP}, k} \Delta t_{k}}
$$

where $i_{\text {meas }, j}$ and $v_{\text {meas }, j}$ are respectively the measured current and voltage of the PV module during the $j^{\text {th }}$ time interval $\Delta t_{j}$, while $i_{\mathrm{MPP}, k}$ and $v_{\mathrm{MPP}, k}$ are the current and voltage corresponding to the MPP of the PV module during the $k^{\text {th }}$ time interval $\Delta t_{k}$.

To accurately track the MPP, a tracker should be able to (1) converge quickly to the region around the MPP, (2) accurately position the operating point at the MPP without oscillating about it and (3) track rapid changes in the MPP without diverging from it, despite noise in the system.

\subsection{Perturb and observe algorithm}

The $\mathrm{P} \& \mathrm{O}$ algorithm is the most commonly employed MPPT algorithm which typically uses the knowledge of the sensed current and voltage values over a couple of samples. It calculates the gradient of measured power $p_{\text {meas }}$ with respect to the applied voltage $v_{\mathrm{PV}}$ between two consecutive iterations at time $t-1$ and $t$. With this knowledge $\mathrm{P} \& \mathrm{O}$ determines the new operating point 
$v_{\mathrm{PV}, t+1}$. The update rule for $v_{\mathrm{PV}, t+1}$ is given by

$$
\begin{aligned}
v_{\mathrm{PV}, t+1} & =v_{\mathrm{PV}, t}+\left(\left(v_{\mathrm{PV}, t}-v_{\mathrm{PV}, t-1}\right) \operatorname{sgn}\left(p_{\text {meas }, t}-p_{\text {meas }, t-1}\right)\right) \\
& =v_{\mathrm{PV}, t}+\Delta v_{\text {step }, t} \operatorname{sgn}\left(p_{\text {meas }, t}-p_{\text {meas }, t-1}\right) .
\end{aligned}
$$

Using this update rule, $\mathrm{P} \& \mathrm{O}$ constantly seeks to correct its operating point leading to superfluous oscillations. When the MPP is unchanging, under static conditions, these superfluous oscillations reduce the algorithm's static efficiency. Under dynamic conditions, when the changes in the MPP are too rapid for the pertubation step size $\Delta v_{\text {step }, t}$ applied at timestep $t$, the operating point of the tracker could diverge from the MPP. At low irradiances where the I-V curves are more flat, the narrow differences in the power levels at consecutive iterations can introduce an error in determining the MPP. If the irradiance $g$ increases, this error would accumulate and lead to a divergence from the MPP.

More advanced implementations of the $\mathrm{P} \& \mathrm{O}$ algorithm are optimized based on the model of the PV system to reduce superfluous oscillations at static conditions if not totally eliminate them and to improve tracking performance under rapidly changing irradiance as well as to enable convergence at low irradiances [33 35]. The perturbation step size $\Delta v_{\text {step }}$ is optimized based on the magnitude and the change in the power of the PV module and hence the operation is based on the model of the PV module. [15, 36]. Moreover by accounting for the noise in the system it lowers the error in predicting the MPP, however, this further increases the complexity of $\mathrm{P} \& \mathrm{O}$.

\section{Bayesian inference based MPP tracker}

The $\mathrm{P} \& \mathrm{O}$ algorithm previously described uses a measured voltage $v_{\text {meas }}, t$ and current $i_{\text {meas }}, t$ value to determine the MPP. In practice, these measurements are affected by noise, and do not correspond exactly to the voltage applied across the PV module, $v_{\mathrm{PV}, t}$ and the current generated by the PV module, $i_{\mathrm{PV}, t}$, respectively. As the noise in the measurements adds uncertainty to these values, they are more correctly represented probabilistically. Modeling this noise as having a Gaussian distribution, the probabilities of the measured voltage and current of the PV module are represented 
as,

$$
\begin{aligned}
p\left(v_{\text {meas }} \mid v_{\mathrm{PV}, t}\right) & =\frac{\mathrm{e}^{-\frac{\left(v_{\text {meas }}-v_{\mathrm{PV}, t}\right)^{2}}{2 \sigma_{v}^{2}}}}{\sqrt{2 \pi \sigma_{v}^{2}}} \\
p\left(i_{\text {meas }} \mid i_{\mathrm{PV}, t}\right) & =\frac{\mathrm{e}^{-\frac{\left(i_{\text {meas }}-i_{\mathrm{PV}, t}\right)^{2}}{2 \sigma_{i}^{2}}}}{\sqrt{2 \pi \sigma_{i}^{2}}} .
\end{aligned}
$$

$\sigma_{i}^{2}$ and $\sigma_{v}^{2}$ are variance of the noise on the current and voltage measurements respectively.

The goal of this paper is to take the inherent noisy nature of the measurements into account by applying probabilistic inference to determine the MPP. To do so, we model a PV module by the directed acyclic graph in Fig. 2. The nodes in this graph represent the variables of the problem, and the directed links the causal relationship between the variables. We use a one-diode model to model the PV module and neglect any impact of varying temperature. Then, the generated current $i_{\mathrm{PV}, t}$ depends on only the applied voltage $v_{\mathrm{PV}, t}$ and on the irradiance $g_{t}$ at the timestep $t$. This implies that the conditional probability of $i_{\mathrm{PV}, t}$ given $v_{\mathrm{PV}, t}$ and $g_{t}$ can be represented by a dirac function at the current $I_{\text {model }}$ described by the model:

$$
p\left(i_{\mathrm{PV}, t} \mid v_{\mathrm{PV}, t}, g_{t}\right)=\delta\left(i_{\mathrm{PV}, t}-\mathrm{I}_{\mathrm{model}}\left(v_{\mathrm{PV}, t}, g_{t}\right)\right)
$$

More accurate models such as a two-diode model or a model that incorporates temperature effects would add complexity [37, 38, while the one-diode model, that we employ, is sufficiently accurate for our application [39, 40].

Additionally, we approximate the irradiance variation between time steps as being a first-order Markov process, meaning that the irradiance $g_{t+1}$ is assumed to depend only on the previous irradiance $g_{t}$ and not on earlier irradiance values. We discuss the conditional probability $p\left(g_{t+1} \mid g_{t}\right)$ in detail in Section 3.1. The measured voltage $v_{\text {meas }, t}$ and current $i_{\text {meas }, t}$ values depend only on the respective voltage $v_{\mathrm{PV}, t}$ and current $i_{\mathrm{PV}, t}$ values of the $\mathrm{PV}$ module, as described by the conditional probabilities of Eqs. (4), (5).

The graph in Fig. 2 represents a so-called Bayesian network [41. Nodes that are disconnected represent variables that are independent of each other. For instance, node $v_{\mathrm{PV}, t}$ is not linked to $g_{t}$ but to $i_{\mathrm{PV}, t}$ and vice versa. Likewise, node $g_{t+1}$ does not influence either $v_{\mathrm{PV}, t}$ or $i_{\mathrm{PV}, t}$ which is made evident by the absence of a direct link between these nodes.

One way to apply probabilistic inference to this graph is by means of Bayesian inference [41. In this technique, the degree of belief in the values of observable parameters is propagated to other 


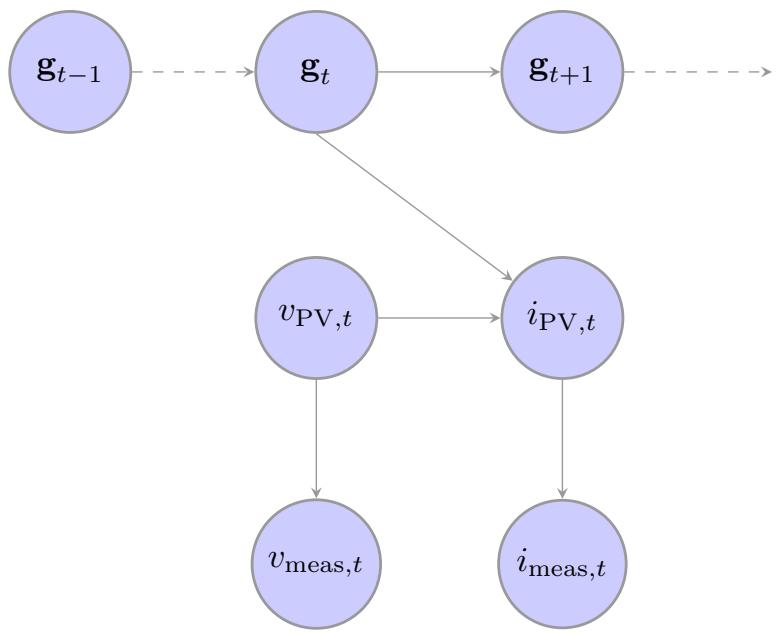

Figure 2: The causal structure of a MPPT problem of a PV module can be captured by a Bayesian network.

relevant parameters, and the belief in the value of the unknown parameter is inferred [42]. For example, Bayesian inference has also been used in weather forecasting based on meteorological data such as clearness index of the sky in predicting the global irradiation. Such a simulator could be a potential operation prediction tool for grid system managers [43. We use Bayesian inference to determine the irradiance at the next timestep based on the current and voltage values of the PV module. In the graph shown in Fig. 2 we solve for the probabilities by propagating the degree of belief in the measured values $i_{\text {meas, } t}$ and $v_{\text {meas, } t}$ as well as the probability distribution $p\left(g_{t-1}\right)$ calculated in the previous iteration, to calculate the probability distribution $p\left(g_{t+1}\right)$. Based on this distribution, we can then calculate the MPP during timestep $t+1$.

From the graph, the probability of the node $g_{t+1}$ can be written as,

$$
\begin{aligned}
& p\left(g_{t+1}\right)=\int p\left(g_{t+1} \mid i_{\text {meas }}, v_{\text {meas }}, g_{t-1}\right) p\left(g_{t-1}\right) \mathrm{d} g_{t-1} \\
&= \iiint \int p\left(g_{t+1}, g_{t}, i_{\mathrm{PV}, t}, v_{\mathrm{PV}, t} \mid i_{\text {meas }, t}, v_{\text {meas }, t}, g_{t-1}\right) \\
& p\left(g_{t-1}\right) \mathrm{d} g_{t-1} \mathrm{~d} g_{t} \mathrm{~d} i_{\mathrm{PV}, t} \mathrm{~d} v_{\mathrm{PV}, t}
\end{aligned}
$$

Note that we use $p\left(g_{t+1}\right)$ as a shorthand notation for the conditional probability distribution of $g_{t+1}$, given the measured current $i_{\text {meas }, t}$ and voltage $v_{\text {meas }, t}$ as well the probability distribution of $g_{t-1}$. To apply probabilistic inference on Bayesian network in a computationally efficient manner, probabilities are typically rewritten as products of conditional probabilities of the form 
$p$ (Effect|Cause) [41]. The reason is that these conditional probabilities are typically well known or easily formulated, whereas likelihoods of the form $p$ (Cause|Effect) are not. The $p$ (Effect|Cause) form can always be obtained by repeatedly applying Bayes' theorem, which relates the likelihood $p$ (Cause|Effect) to $p$ (Effect|Cause) and the prior probabilities $p$ (Cause) and $p$ (Effect) [4] according to:

$$
p(\text { Cause } \mid \text { Effect })=\frac{p(\text { Effect } \mid \text { Cause }) p(\text { Cause })}{p(\text { Effect })} .
$$

By taking into account the dependencies captured by the graph in Fig. 2 , we can rewrite the conditional probability in Eq. (8) as:

$$
\begin{aligned}
p\left(g_{t+1}, g_{t}, i_{\mathrm{PV}, t}, v_{\mathrm{PV}, t} \mid i_{\text {meas }, t}, v_{\text {meas }, t}, g_{t-1}\right) \\
=p\left(g_{t+1} \mid g_{t}\right) p\left(g_{t}, i_{\mathrm{PV}, t}, v_{\mathrm{PV}, t} \mid i_{\text {meas }, t}, v_{\text {meas }, t}, g_{t-1}\right) \\
=p\left(g_{t+1} \mid g_{t}\right) p\left(i_{\mathrm{PV}, t} \mid i_{\text {meas }, t}, g_{t}, v_{\mathrm{PV}, t}\right) p\left(g_{t} \mid g_{t-1}\right) p\left(v_{\mathrm{PV}, t} \mid v_{\text {meas }, t}\right) .
\end{aligned}
$$

In the above equation, the conditional probabilities of $i_{\mathrm{PV}, t}$ and $v_{\mathrm{PV}, t}$ can be rewritten using Bayes' Theorem of Eq. (9) as:

$$
\begin{aligned}
p\left(i_{\mathrm{PV}, t} \mid i_{\text {meas }, t}, g_{t}, v_{\mathrm{PV}, t}\right) & =\frac{p\left(i_{\text {meas }, t} \mid i_{\mathrm{PV}, t}, g_{t}, v_{\mathrm{PV}, t}\right) p\left(i_{\mathrm{PV}, t} \mid g_{t}, v_{\mathrm{PV}, t}\right)}{p\left(i_{\text {meas }, t}\right)} \\
& =\frac{p\left(i_{\text {meas }, t} \mid i_{\mathrm{PV}, t}\right) p\left(i_{\mathrm{PV}, t} \mid g_{t}, v_{\mathrm{PV}, t}\right)}{p\left(i_{\text {meas }, t}\right)} \\
p\left(v_{\mathrm{PV}, t} \mid v_{\text {meas }, t}\right) & =\frac{p\left(v_{\text {meas }, t} \mid v_{\mathrm{PV}, t}\right) p\left(v_{\mathrm{PV}, t}\right)}{p\left(v_{\text {meas }, t}\right)}
\end{aligned}
$$

where the probabilities $p\left(i_{\text {meas }, t}\right), p\left(v_{\text {meas }, t}\right)$, and $p\left(v_{\mathrm{PV}, t}\right)$ are the prior probabilities of the corresponding parameters. Substituting Eq. (11) into Eq. (8), leads to:

$$
\begin{aligned}
& p\left(g_{t+1}\right)=\alpha \iiint \int p\left(g_{t+1} \mid g_{t}\right) p\left(i_{\text {meas }, t} \mid i_{\mathrm{PV}, t}\right) p\left(i_{\mathrm{PV}, t} \mid g_{t}, v_{\mathrm{PV}, t}\right) \\
& p\left(v_{\text {meas }, t} \mid v_{\mathrm{PV}, t}\right) p\left(g_{t} \mid g_{t-1}\right) p\left(g_{t-1}\right) \mathrm{d} g_{t-1} \mathrm{~d} g_{t} \mathrm{~d} i_{\mathrm{PV}, t} \mathrm{~d} v_{\mathrm{PV}, t}
\end{aligned}
$$

where we absorbed the prior probabilities in the normalization factor $\alpha$. As it is common in Bayesian inference, $\alpha$ is not explicitly calculated but taken into account by normalizing the result of the inference, i.e., by normalizing the sum of the probabilities $p\left(g_{t+1}\right)$ to 1 at the end of the calculation. 
By susbstituting Eqs. (4), (5) in Eq. (14) we obtain,

$$
\begin{array}{r}
p\left(g_{t+1}\right)=\alpha \iiint \int p\left(g_{t+1} \mid g_{t}\right) \mathrm{e}^{-\frac{\left(i_{\mathrm{PV}, t}-i_{\text {meas }, t}\right)^{2}}{2 \sigma_{i}^{2}}} \delta\left(i_{\mathrm{PV}, t}-\mathrm{I}_{\text {model }}\left(g_{t}, v_{\mathrm{PV}, t}\right)\right) \\
\mathrm{e}^{-\frac{\left(v_{\mathrm{PV}, t}-v_{\text {meas }, t}\right)^{2}}{2 \sigma_{v}^{2}}} p\left(g_{t} \mid g_{t-1}\right) p\left(g_{t-1}\right) \mathrm{d} g_{t-1} \mathrm{~d} g_{t} \mathrm{~d} i_{\mathrm{PV}, t} \mathrm{~d} v_{\mathrm{PV}, t} \\
=\alpha \int \mathrm{d} g_{t} p\left(g_{t+1} \mid g_{t}\right) p_{0}\left(g_{t}\right) \\
\int \mathrm{e}^{-\frac{\left(\mathrm{I}_{\text {model }}\left(g_{t}, v_{\mathrm{PV}, t}\right)-i_{\text {meas }, t}\right)^{2}}{2 \sigma_{i}^{2}}} \mathrm{e}^{-\frac{\left(v_{\mathrm{PV}, t}-v_{\text {meas }, t}\right)^{2}}{2 \sigma_{v}^{2}}} \mathrm{~d} v_{\mathrm{PV}, t}
\end{array}
$$

Here, we introduced $p_{0}\left(g_{t}\right)$ as the probability distribution of $g_{t}$ that was calculated during the previous timestep. The newly calculated $p\left(g_{t+1}\right)$ is used as the prior probability $p_{0}\left(g_{t+1}\right)$ in the next iteration while calculating $p\left(g_{t+2}\right)$. With the knowledge of the irradiance for the next timestep, the BI-MPPT computes the new operating point as

$$
v_{\mathrm{PV}, t+1}=\int v_{M P P}\left(g_{t+1}\right) p\left(g_{t+1}\right) \mathrm{d} g_{t+1}
$$

where $v_{M P P}\left(g_{t+1}\right)$ is the modelled MPP for the irradiance $g_{t+1}$, determined by also using the same one-diode model of the PV module.

\subsection{Conditional probability of $g_{t+1}$ given $g_{t}$}

To find $p\left(g_{t+1} \mid g_{t}\right)$, we gathered irradiance measurement data over several days of varying weather conditions. In the measurement data we observed that the probability of a difference in irradiance $g_{t+1}-g_{t}$ occurring is independent of the irradiance value $g_{t}$. Based on this observation, we approximate the conditional probability $p\left(g_{t+1} \mid g_{t}\right)$ as being only a function of the difference in irradiance between two consecutive time steps:

$$
p\left(g_{t+1} \mid g_{t}\right)=p\left(g_{t+1}-g_{t}\right)
$$

Fig. 3 shows the probability distribution $p\left(g_{t+1}-g_{t}\right)$ observed in our measurement data. It also shows a fitted curve that is a combination of piecewise linear and logarithmic fits. We used this fitted curve to approximate $p\left(g_{t+1}-g_{t}\right)$ in our simulations. 
Based on Eq. (18), Eq. (16) can be re-written as:

$$
\begin{aligned}
p\left(g_{t+1}\right)= & \alpha \int \mathrm{d} g_{t} p\left(g_{t+1}-g_{t}\right) p_{0}\left(g_{t}\right) \\
& \int_{v} \mathrm{e}^{-\frac{\left(\mathrm{I} \text { model }\left(g_{t}, v_{\mathrm{PV}, t}\right)-i_{\text {meas }, t}\right)^{2}}{2 \sigma_{i}^{2}}} \mathrm{e}^{-\frac{\left(v_{\mathrm{PV}, t}-v_{\text {meas }, t}\right)^{2}}{2 \sigma_{v}^{2}}} \mathrm{~d} v_{\mathrm{PV}, t} .
\end{aligned}
$$

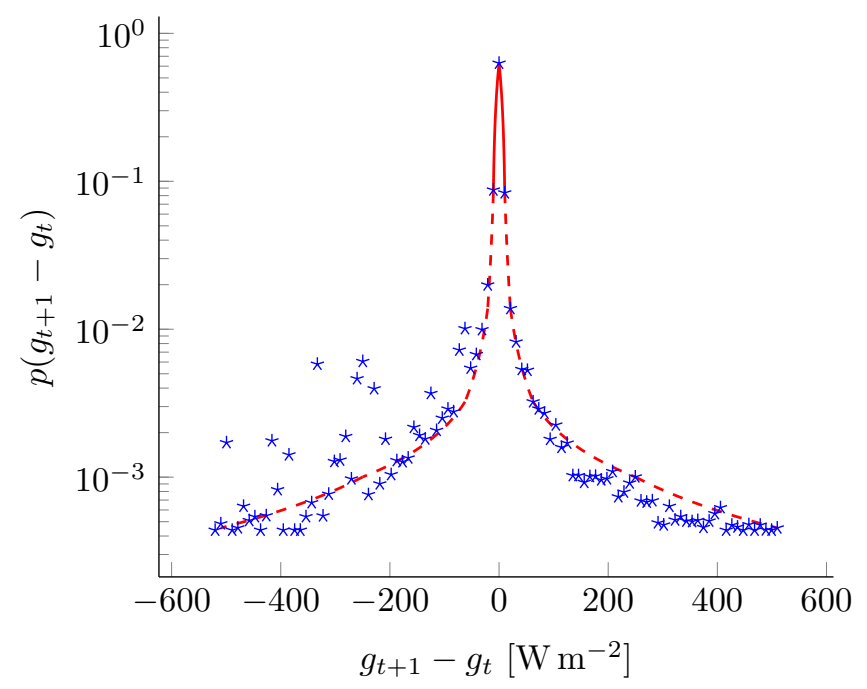

Figure 3: Linear (- $)$ and logarithmic (- - -) fit of averaged transition probabilities of irradiance $g_{t+1}$ at time $t+1$, given irradiance $g_{t}$ at time $t$, based on measurement data $(\star)$ simplifies the conditional probability $p\left(g_{t+1} \mid g_{t}\right)$ to $p\left(g_{t+1}-g_{t}\right)$ for any $p\left(g_{t}\right)$.

Here, the voltage integral can be numerically calculated and the irradiance integral can be evaluated as a convolution. For every iteration, given the measured current and voltage values, the irradiance value for the next timestep is used to calculate the I-V curve of the given PV module from which the voltage value corresponding to the MPP is determined, thus enabling tracking of the MPP.

\subsection{Implementation of BI-MPPT}

In implementing the algorithm, we have used equidistant discretized array of $g$ and $v_{\mathrm{PV}}$ values. For our implementation, $g$ array has been discretized into $N_{g}=32$ elements and $v_{\mathrm{PV}}$ array into 128 elements. Optimization of these arrays shall be addressed in future implementation. In Fig. 4 we provide the flowchart of our implementation of the BI-MPPT algorithm. 


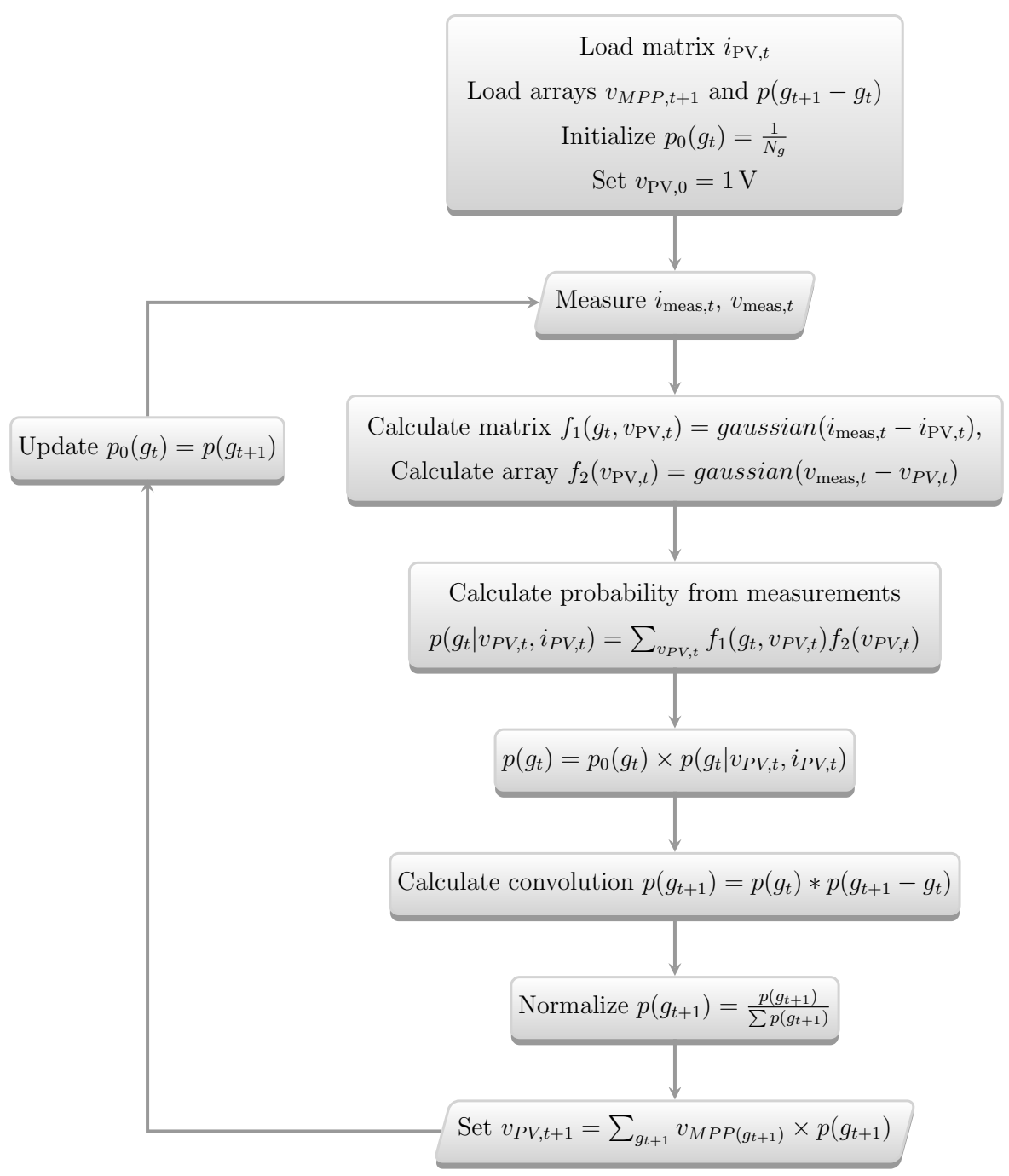

Figure 4: Flowchart of a BI-MPPT implementation.

First, the matrix $i_{\mathrm{PV}, t}=I_{\text {model }}\left(g_{t}, v_{\mathrm{PV}, t}\right)$ and the array $v_{\mathrm{MPP}}\left(g_{t+1}\right)$ are loaded based on the cell model. The array $p\left(g_{t+1}-g_{t}\right)$ is loaded based on the approximate conditional probability of Fig. 3 The probability $p_{0}\left(g_{t}\right)$ is initialized at uniform probabilities and the operating voltage for the first iteration $v_{\mathrm{PV}, 0}$ is set to a chosen value. This initial operating voltage has no impact on the results.

The tracker then operates in a loop that starts by measuring $i_{\text {meas }, t}$ and $v_{\text {meas }, t}$ of the present timestep. Eq. 16 is then evaluated using 5 steps:

1. the Gaussian probabilities of $i_{\text {meas }, t}-i_{\mathrm{PV}, t}$ and $v_{\text {meas }, t}-v_{\mathrm{PV}, t}$ are evaluated which are denoted 
as the functions $f_{1}\left(g_{t}, v_{\mathrm{PV}, t}\right)$ and $f_{2}\left(v_{\mathrm{PV}, t}\right)$ respectively,

2. the two Gaussian probabilities are multiplied and summed over $v_{\mathrm{PV}, t}$, leading to the probability $p\left(g_{t} \mid v_{\mathrm{PV}, t}, i_{\mathrm{PV}, t}\right)$ based on the present measurement,

3. the probability is combined with the probability $p_{0}\left(g_{t}\right)$ from previous measurements to calculate the total probability $p\left(g_{t}\right)$,

4. the total probability is convoluted with the transition probability to calculate the probability $p\left(g_{t+1}\right)$ of the irradiance at the next timestep,

5. this probability is normalized to take the normalization factor $\alpha$ into account.

Using the calculated probability $p\left(g_{t+1}\right)$, the operating voltage $v_{\mathrm{PV}, t+1}$ is calculated for the next timestep by averaging over $v_{M P P}\left(g_{t+1}\right)$ according to Eq. 17. The probability $p_{0}\left(g_{t}\right)$ is then updated with $p\left(g_{t+1}\right)$, and the next measurement is made.

\section{Performance analysis}

The performance of the proposed BI-MPPT is assessed by operating it at static and dynamic illumination using an indoor experimental set-up, and to outdoor measured I-V curves under rapidly changing irradiance conditions. The algorithm is also compared to the optimized $\mathrm{P} \& \mathrm{O}$ algorithm discussed in Section 2.1. As previously explained, both the BI-MPPT and the optimized P\&O are model-based trackers, with the latter being a gradient descent technique for which the step size is optimized based on a model of the PV module [15]. Both the trackers use identical models of the $\mathrm{PV}$ modules used in the indoor experiment or outdoor measurements as appropriate.

\subsection{Experimental setup}

The in-house solar emulator - used to demonstrate the operation of the MPPTs in the experimentsconsists of an array of forty-six halogen lamps whose illumination is controllable with a power supply with heat sinks, an irradiance sensor, and a reconfigurable PV module built with polycrystalline Maxis solar cells 44] which is shown in Fig. 5(a)] A set of reconfigurable switches have been added between each pair of the cells [45. Ten series-connected cells of the PV module are connected to an 


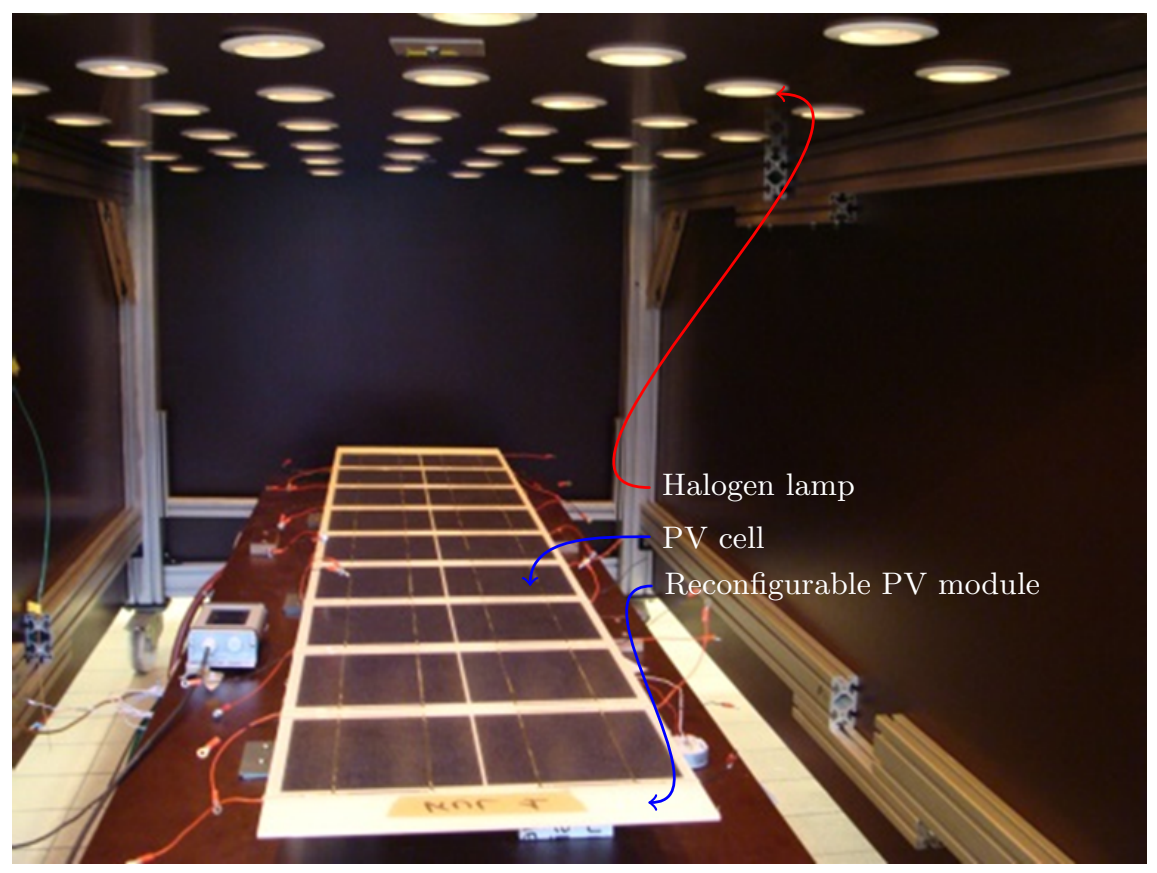

(a)

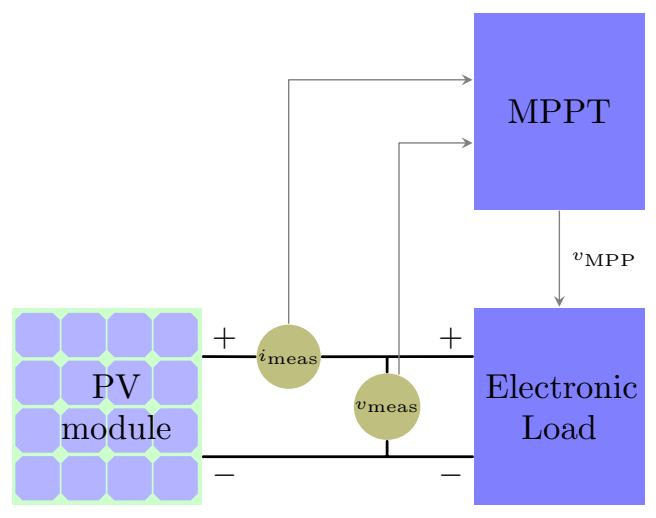

(b)

Figure 5: (a) Experimental setup consists of an array of 46 halogen lamps and 10 of the reconfigurable polycrystalline Maxis solar cells connected in series. (b) The setup is operated by an electronic load whose operating voltage $v_{\text {MPP }}$ is set by a MPPT algorithm.

electronic load which is in turn interfaced with Matlab [46. This configuration forms a hardwarein-loop setup where the tracking algorithms are developed in Matlab control the operation of the PV module. The operating point is set by the electronic load based on the measured $v_{\text {meas }}$ and 
$i_{\text {meas }}$ (see Fig. 5(b) . This setup is also capable of measuring the I-V characteristics of the module and the operating current and voltage of individual cells. The settling time of this system, from $50 \mathrm{~ms}$ up to $1 \mathrm{~s}$, is mainly due to the electronic load.

To find the tracker efficiency during the experiments, we estimate the MPP values in the denominator of Eq. (1) based on the characterization of the PV module for a set of illumination values. For each value, we measure the MPP under static illumination conditions. Based on these measurements we compile a look-up table that specifies the MPP for each illumination value.We estimate the MPP for a given illumination value by using a piecewise linear approximation based on this table. The effects of temperature are neglected in composing the look-up table to avoid a large and complex dataset. Although module temperature depends on ambient temperature, heat transfer, illumination and operating point, the operation times in the laboratory experiments are sufficiently short for any change in the ambient temperature and likewise the cell temperature to be neglected 47. In case of the convergence experiments in Section 4.2, we also use a second method to estimate the MPP. In these experiments, we apply a step in illumination to the PV module.

For the second method, we measure the I-V curves at the beginning and end of the experiment, and note for each the corresponding MPP. We use the MPP from the beginning (end) I-V curve as the MPP at the time samples before (after) the step is applied. Since we are experimenting with a step between two static irradiance values, all the I-V curves are expected to lie close to the corresponding measured I-V curve.

\subsection{Convergence and static operation}

In this experiment we test for convergence by creating a step in the illumination, and thereafter, test for operation at static MPP conditions by not changing the illumination. The MPPTs are operated for the first 10 iterations with the halogen lamps turned off, mimicking $0 \mathrm{~W} \mathrm{~m}^{-2}$ and at the instant of the tenth iteration the the halogen lamps are powered to provide approximately $50 \mathrm{~W} \mathrm{~m}^{-2}$. The illumination levels are only approximately given as the light source is not a closed-loop system. When the halogen lamps in our setup heat up, the power supply would regulate the power to the lamps which can cause small differences in the desired illumination.

The operating points of the proposed BI-MPPT are shown in Fig. 6(a) to correspond well with the MPP values. For the BI-MPPT, the operating point converges within one iteration after applying the illumination step. Note that this shows that BI-MPPT can achieve tracking without 


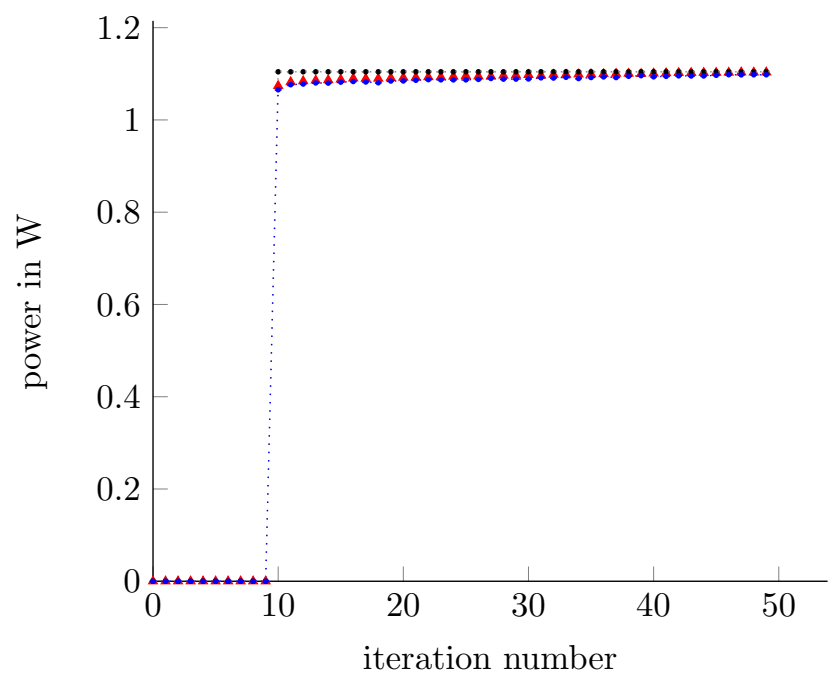

(a)

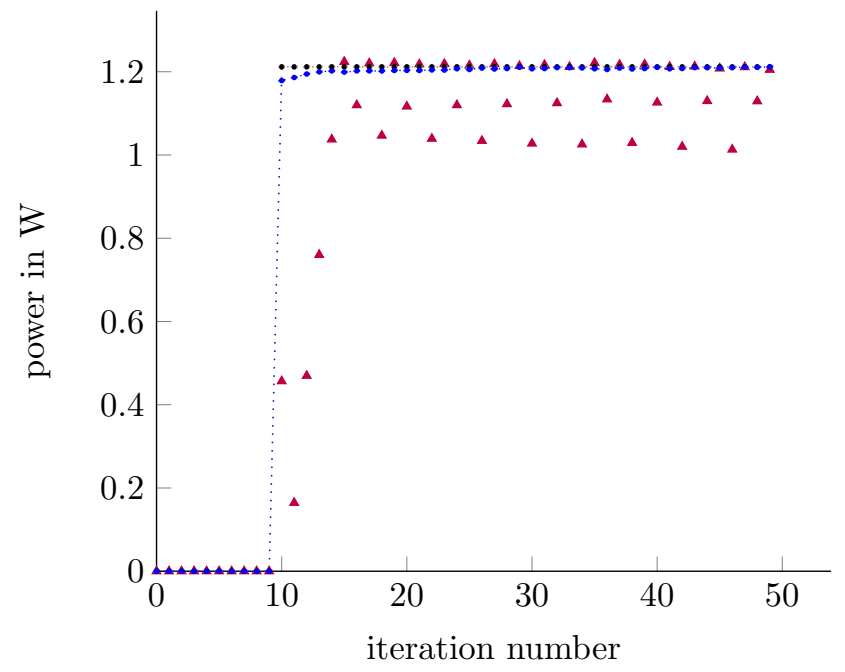

(b)

Figure 6: Response of the trackers $(\boldsymbol{\Delta})$ is compared to the expected MPP values by method one using look-up table(.....) and method two using direct MPP measurements (.....): (a) bayesian inference based MPPT algorithm and (b) P\&O algorithm at static irradiance in the lab when illumination is stepped up to approximately $50 \mathrm{~W} \mathrm{~m}^{-2}$.

a systematic error while using a simplified model of the PV module. In contrast, optimized P\&O takes five iterations to converge in Fig. 6(b) which is inherent to its hill-climbing nature. The decrease in the MPPT efficiency is dependent on both the convergence and the amplitude of its 
oscillation at steady-state. Decreasing the amplitude of the oscillation would slow the convergence of the tracker [48]. The overall efficiency of BI-MPPT is $99.9 \%$ and $99.4 \%$ when respectively using the first and second methods of estimating the real MPP; whereas the optimized $\mathrm{P} \& \mathrm{O}$ tracker realizes efficiencies of $89.5 \%$ and $89 \%$. This loss in efficiency of the optimized P\&O is due its oscillating nature. It is indeed well established that the problem of $\mathrm{P} \& \mathrm{O}$ is its oscillation about the MPP on converging to it at steady-state [49]. Oscillations are absent in the BI-based tracker due to its model-based approach which helps in faster convergence. For the experiments performed, BI-MPPT shows an improvement in the energy efficiency of 10 percentage points over that of the optimized P\&O tracker.

\subsection{Dynamic performance}

In the first experiment of testing the dynamic performance of the MPPT, the illumination is varied according to a pre-defined profile consisting of a step-up and -down in illumination, followed by a ramp-up and -down. There is a difference of $5 \mathrm{~W} \mathrm{~m}^{-2}$ between any two sample points in the ramp. Ramp profiles often appear in outdoor conditions. In addition to this reason, MPPTs are difficult at tracking such a constant change in a single direction [50, 51, thus incurring losses. It is therefore important to test MPPTs on ramp profiles.

The response of BI-MPPT is shown in comparison to the estimated MPP values in Fig. 7(a), The overall dynamic efficiency is $97.4 \%$ and that during the ramps is $94.5 \%$. The response of the optimized P\&O tracker is shown in Fig. 7(b), whose overall dynamic efficiency is $87 \%$ and that during the ramps $84.6 \%$. The BI-MPPT is able to track monotonous increase or decrease in illumination while $\mathrm{P} \& \mathrm{O}$ suffers from large fluctuations and is unable to track the fast changes as it requires a larger transient tracking time 35. The large fluctuations of $\mathrm{P} \& \mathrm{O}$ are mainly due to the large errors introduced by its perturbations [22] which also causes the slower convergence of the $\mathrm{P} \& \mathrm{O}$ algorithm at low irradiance conditions.

In the second experiment shown in Fig. 8(a), we consider the response of the BI-MPPT to random variation in low illumination. The BI-MPPT in this experiment realizes a dynamic tracking efficiency of $97.6 \%$ which is consistent with the dynamic efficiency computed in the previous experiment. The BI-MPPT demonstrates a quick response to illumination changes by converging to the MPP value during a sudden increase as well as a sudden decrease in the illumination value. This can be seen at the first four illumination steps within $25 \mathrm{~s}$ in Fig. 8(a). The BI-MPPT is also 


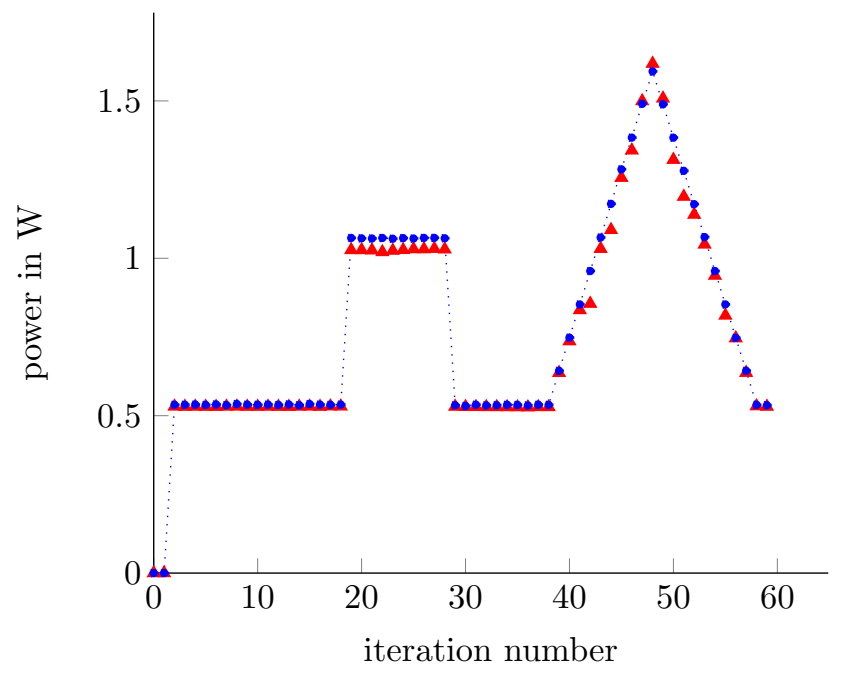

(a)

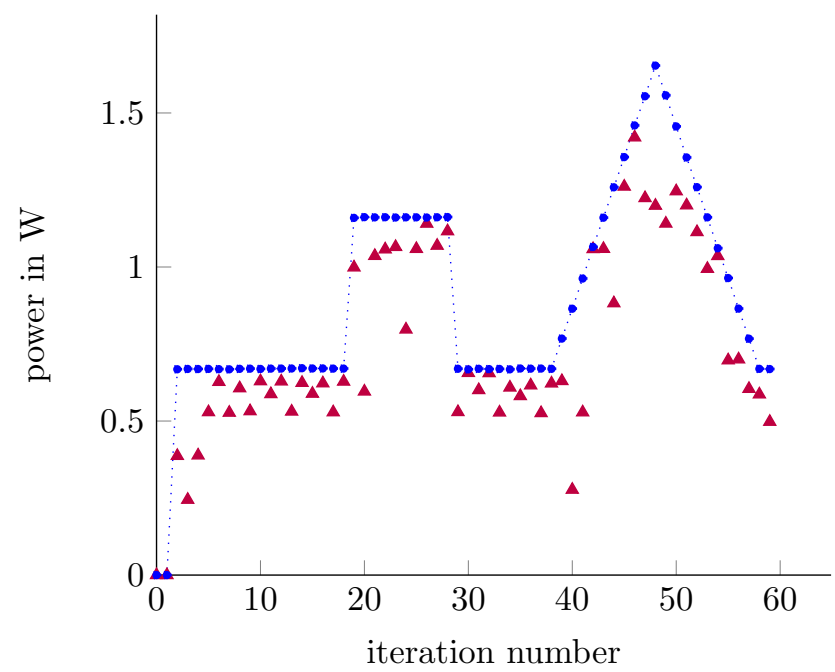

(b)

Figure 7: (a) The response of the BI-MPPT algorithm ( $\Delta$ ) and (b) the response of the P\&O algorithm ( $\Delta)$ to a dynamic illumination profile are compared with the fitted MPP values $(\cdots \cdots)$.

able to track a slower rate of change in illumination with errors smaller than $13.6 \%$, as can be seen between $20 \mathrm{~s}$ to $40 \mathrm{~s}$. The overall dynamic efficiency of the BI-MPPT is shown alongside its evolution in Fig. 8(b),

Our indoor dynamic analysis shows that at low irradiance conditions the BI-MPPT is able 


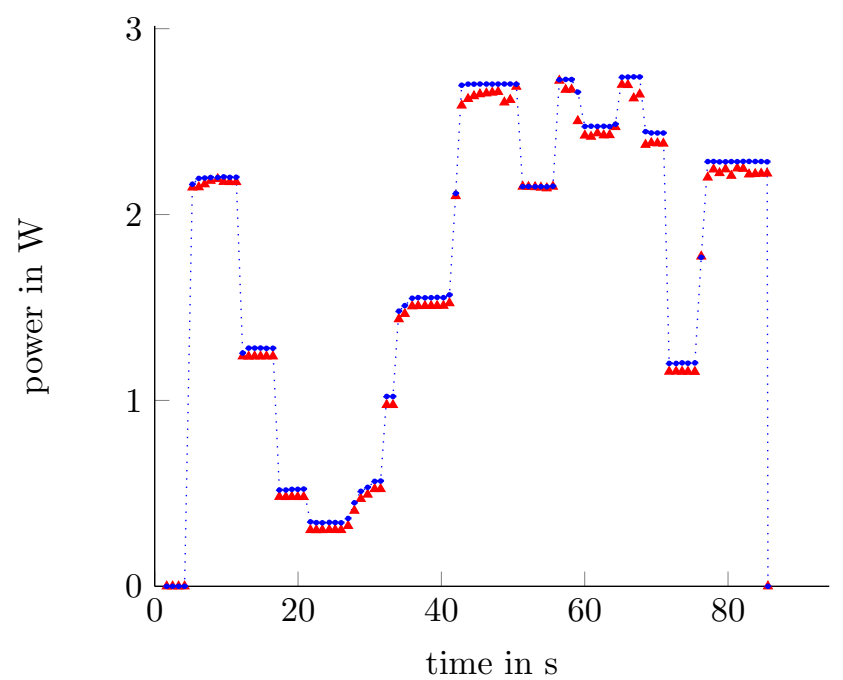

(a)

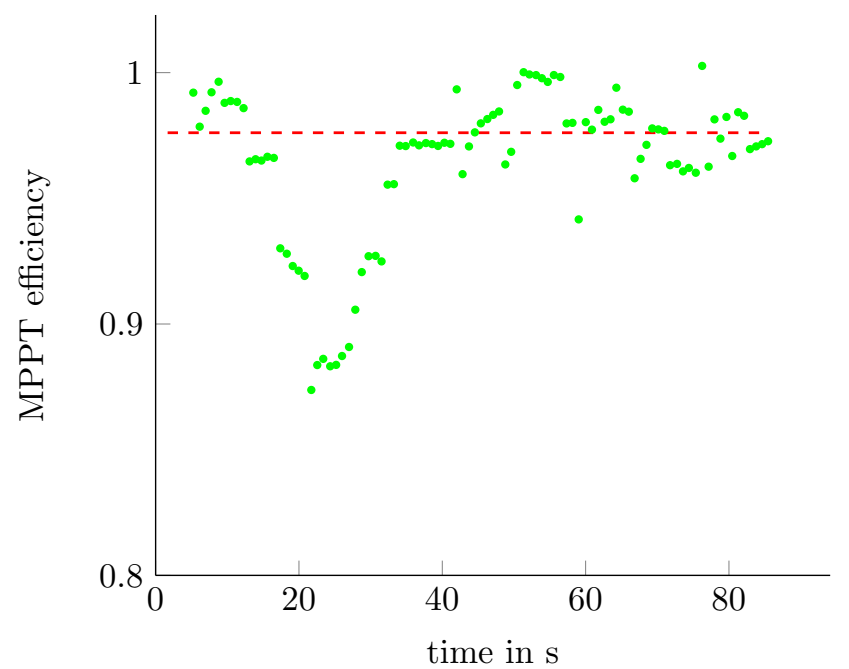

(b)

Figure 8: (a) The response of the BI-MPPT algorithm to dynamic illumination profile $(\Delta)$ is compared with the fitted MPP values $(\cdots \cdots)$ and $(\mathrm{b})$ shows the evolution of the MPPT efficiency over the iterations $(\bullet)$ as well as the overall dynamic efficiency (-- -).

to converge fast to- and track the MPP, for (1) abrupt changes in illumination in the form of steps or, (2) monotonous changes in the form of ramps, (3) as well for slow and random changes in illumination. The overall poorer performance of the optimized $\mathrm{P} \& \mathrm{O}$ is attributed to the low 
irradiance conditions [4, 5]. We have also showed that, in contrast to the optimized P\&O, the proposed tracker performs well for both increasing and decreasing illumination profiles.

\subsection{Analysis of the tracker based on meteorological data}

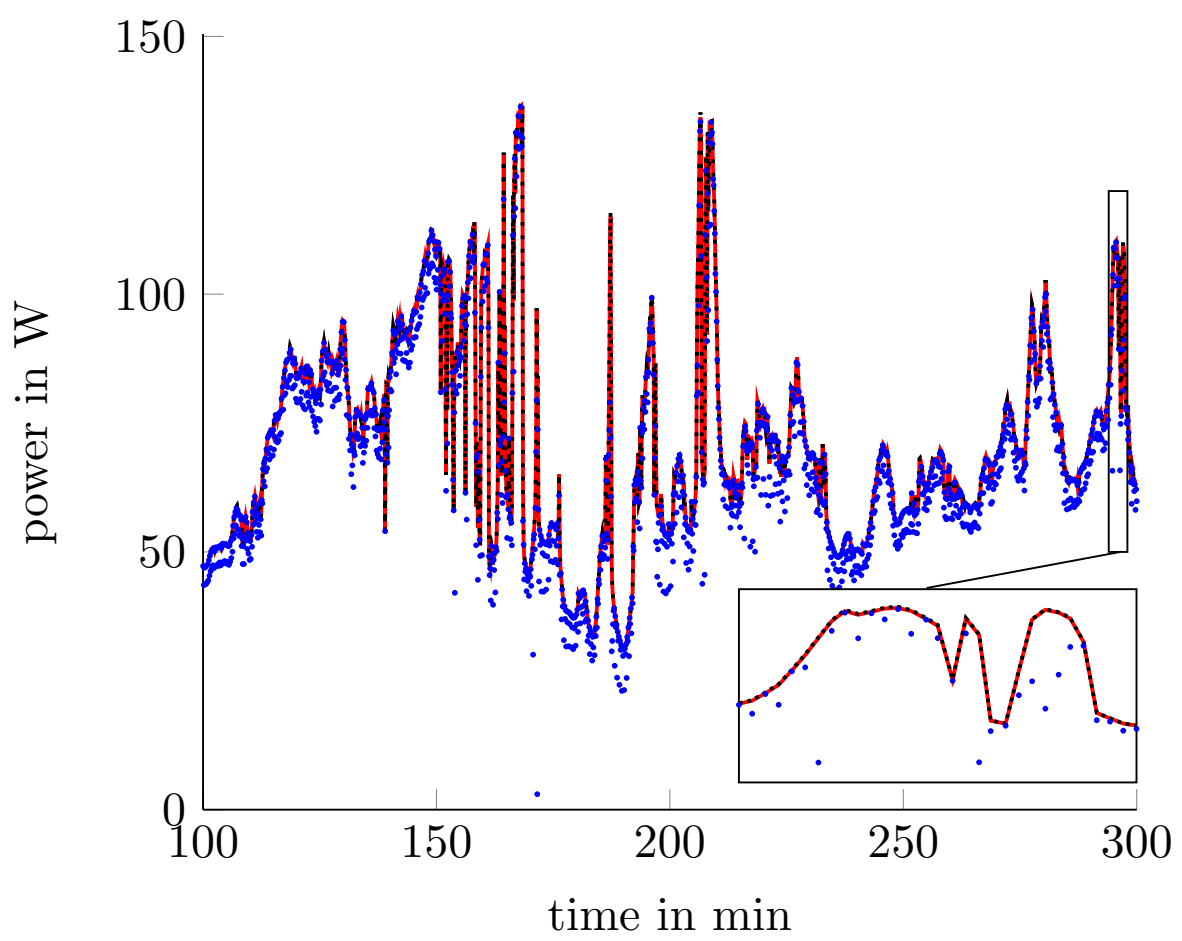

Figure 9: BI-MPPT algorithm (-) performs better than the optimized P\&O $(\bullet)$ when compared to the MPP values $(\cdots \cdots)$, at dynamically changing medium and high irradiance levels based on measurement data.

Finally, we have also analyzed the performance of the trackers with simulations based on measured meteorological data. To do so, we have measured I-V curves about every 6 seconds from PHX-160 polycrystalline PV modules of Phoenix solar on several days between March and April 2014. We use these curves to demonstrate the tracker under outdoor conditions and scenarios that cannot be generated in the laboratory, which in our case would be medium and high irradiance conditions [52]. The I-V curves are fed into the Matlab-based PV simulation tool [53] with which the MPPTs are tested. As an example, the responses of the trackers for a part of a day are shown in Fig. 9 .

Being model based, the BI-MPPT bases its estimation of the operating point on the noisy 


\begin{tabular}{llc}
\hline & BI-MPPT[\%] & P\&O[\%] \\
\hline Low irradiance indoor experiment & & \\
\hline static & 99.9 & 89.5 \\
dynamic: step, ramp & 97.4 & 87 \\
random variations & 97.6 & \\
\hline Measurement data & & \\
\hline Day with sparse/isolated rapid changes & 99.08 & 96.16 \\
Day with intermittent rapid changes & 99.61 & 95.61 \\
Day with frequent rapid changes & 98.92 & 94.85 \\
\hline
\end{tabular}

Table 1: BI-MPPT tracking efficiency is consistently high for a broad range of irradiance conditions.

samples and the relationship established between two consecutive irradiances brought out by the transition probability in Sec. 3.1. Therefore, BI-MPPT is able to track any magnitude of irradiance changes and is unaffected by the speed of the irradiance change as shown in the inset in Fig. 9. The optimized $\mathrm{P} \& \mathrm{O}$ on the other hand, is able to track large differences in the irradiance so long as the irradiance is more or less constant following this change. Being a gradient descent algorithm, $\mathrm{P} \& \mathrm{O}$ requires a few additional iterations to converge after tracking large differences in irradiance. As a consequence, $\mathrm{P} \& \mathrm{O}$ is unable to track rapidly changing irradiance as good as BI-MPPT.

For an entire day, the dynamic efficiency for BI-MPPT is $99.61 \%$ and that of optimized P\&O, which performs well at high irradiance, is $95.61 \%$. BI-MPPT consistently performs well as summarized in Tab. 1. This indicates that the proposed BI-MPPT is able to track the MPP under a broad range of realistic irradiance conditions.

\section{Conclusion}

We proposed a novel maximum power point tracker called BI-MPPT which uses Bayesian inference to estimate the irradiance from the measured current and voltage operating values. The proposed 
BI-MPPT is a model-based tracker and inherently accounts for random noise in the system due to its probabilistic approach. The tracker also uses the relationship between two consecutive irradiance values as a conditional probability, which we have derived from outdoor measurement data. We applied this conditional probability and the noisy current and voltage measurements to Bayes' rule. The result of this operation was an irradiance distribution which when combined with the MPPs of the PV module, obtained from its simple model, gives the operating voltage of the next iteration.

We compared the BI-MPPT to a tracker based on the P\&O algorithm with an optimized perturbation step size. We evaluated both the trackers on an in-house built hardware-in-loop solar emulator. Here, we tested the proposed BI-MPPT for convergence by applying a step in the illumination. The overall efficiency of the proposed BI-MPPT was shown to be greater than $99 \%$, and also to perform consistently better than the optimized P\&O by about 10 p.p. We showed that the BI-MPPT was able to track step-up, step-down and ramp profiles well, with very small transient tracking time. We also showed that BI-MPPT performed equally well on ascending and descending ramps unlike the $\mathrm{P} \& \mathrm{O}$. When slow changes were applied at low illumination, the BI-MPPT was able to track with occasional errors that were less than 13 p.p. The overall dynamic efficiency of the BI-MPPT calculated in both the low illumination experiments was at the least $97.4 \%$.

Using outdoor measurement data we subjected both the trackers to identical environmental conditions and compared the performances at medium and high irradiances. BI-MPPT was able to track the different magnitudes of change in irradiance which the optimized $\mathrm{P} \& \mathrm{O}$, being a gradient descent technique, sometimes failed to track. The dynamic efficiency of the BI-MPPT, based on measurement data varied between 98.92 and $99.61 \%$ for the three different days and were about 4 p.p more than that of $\mathrm{P} \& \mathrm{O}$. Using only a simple one-diode model of the PV modules, BI-MPPT performed better than the optimized $\mathrm{P} \& \mathrm{O}$.

Our results show that the proposed BI-MPPT outperformed the conventional optimized P\&O algorithm under a wide variety of operating conditions. In our experiments, BI-MPPT used a simple one-diode model of the PV module which is indicative of the proposed tracker not requiring a detailed model in practice. BI-MPPT was able to track both static and dynamic irradiance variations, and under low, as well as medium and high irradiance conditions, making it a promising candidate for many emerging PV applications. 


\section{Acknowledgment}

The authors acknowledge the support of the SMART PV project funded by IWT-SBO grant number 110025 .

\section{References}

[1] D. L. King, J. A. Kratochvil, W. E. Boyson, Measuring solar spectral and angle-of-incidence effects on photovoltaic modules and solar irradiance sensors, in: Conference Record of the Twenty Sixth IEEE Photovoltaic Specialists Conference, 1997, pp. 1113-1116. doi:10.1109/ PVSC.1997.654283

[2] J.-C. Wang, Y.-L. Su, J.-C. Shieh, J.-A. Jiang, High-accuracy maximum power point estimation for photovoltaic arrays, Solar Energy Materials and Solar Cells 95 (3) (2011) 843-851. doi: https://doi.org/10.1016/j.solmat.2010.10.032.

[3] M. Jantsch, M. Real, H. Häberlin, C. Whitaker, K. Kurokawa, G. Blässer, P. Kremer, C. Verhoeve, Measurement of PV maximum power point tracking performance, in: 14th European Photovoltaic Solar Energy Conference and Exhibition, 1997.

[4] J. Ahmed, Z. Salam, An improved perturb and observe (P\&O) maximum power point tracking (MPPT) algorithm for higher efficiency, Applied Energy 150 (2015) 97 - 108. doi:http: //dx.doi.org/10.1016/j.apenergy.2015.04.006.

[5] D. P. Hohm, M. E. Ropp, Comparative study of maximum power point tracking algorithms, Progress in Photovoltaics: Research and Applications 11 (1) (2003) 47-62. doi:10.1002/pip. 459 .

[6] M. F. N. Tajuddin, M. S. Arif, S. M. Ayob, Z. Salam, Perturbative methods for maximum power point tracking (MPPT) of photovoltaic (PV) systems: a review, International Journal of Energy Research 39 (9) (2015) 1153-1178.

[7] N. Femia, G. Petrone, G. Spagnuolo, M. Vitelli, A new analog MPPT technique: TEODI, Progress in photovoltaics: research and applications 18 (1) (2010) 28-41. 
[8] Y. Jiang, J. A. A. Qahouq, A. Hassan, E. Abdelkarem, M. Orabi, Load current based analog MPPT controller for PV solar systems, in: Twenty-Seventh Annual IEEE Applied Power Electronics Conference and Exposition (APEC), IEEE, 2012, pp. 911-914.

[9] S.-Y. Wang, H.-H. Wu, C.-L. Wei, An Integrated Boost Converter with Maximum Power Point Tracking for Solar Photovoltaic Energy Harvesting, in: International Symposium on VLSI Design, Automation and Test (VLSI-DAT), IEEE, 2014, pp. 1-4.

[10] R. Leyva, P. Artillan, C. Cabal, B. Estibals, C. Alonso, Dynamic performance of maximum power point tracking circuits using sinusoidal extremum seeking control for photovoltaic generation, International Journal of Electronics 98 (4) (2011) 529-542.

[11] R. C. Hsu, C.-T. Liu, W.-Y. Chen, H.-I. Hsieh, H.-L. Wang, A Reinforcement LearningBased Maximum Power Point Tracking Method for Photovoltaic Array, International Journal of Photoenergy 2015.

[12] M. B. Shadmand, R. S. Balog, H. Abu-Rub, Model predictive control of PV sources in a smart DC distribution system: Maximum power point tracking and droop control, IEEE Transactions on Energy Conversion 29 (4) (2014) 913-921.

[13] Y. Liu, H. Xin, Z. Wang, T. Yang, Power control strategy for photovoltaic system based on the Newton quadratic interpolation, Renewable Power Generation 8 (6) (2014) 611-620.

[14] P. E. Kakosimos, A. G. Kladas, Implementation of photovoltaic array MPPT through fixed step predictive control technique, Renewable Energy 36 (9) (2011) 2508-2514.

[15] N. Femia, G. Petrone, G. Spagnuolo, M. Vitelli, Optimization of perturb and observe maximum power point tracking method, IEEE Transactions on Power Electronics 20 (4) (2005) 963-973.

[16] G.-C. Hsieh, H.-I. Hsieh, C.-Y. Tsai, C.-H. Wang, Photovoltaic power-increment-aided incremental-conductance mppt with two-phased tracking, IEEE Transactions on Power Electronics 28 (6) (2013) 2895-2911.

[17] S. Saravanan, N. R. Babu, Maximum power point tracking algorithms for photovoltaic systema review, Renewable and Sustainable Energy Reviews 57 (2016) 192-204. 
[18] N. Khaehintung, T. Wiangtong, P. Sirisuk, FPGA Implementation of MPPT Using Variable Step-Size P\&O Algorithm for PV Applications, in: 2006 International Symposium on Communications and Information Technologies, 2006, pp. 212-215. doi:10.1109/ISCIT.2006. 340033 .

[19] A. K. Abdelsalam, A. M. Massoud, S. Ahmed, P. N. Enjeti, High-performance adaptive perturb and observe mppt technique for photovoltaic-based microgrids, IEEE Transactions on Power Electronics 26 (4) (2011) 1010-1021. doi:10.1109/TPEL.2011.2106221.

[20] G. Petrone, C. A. Ramos-Paja, G. Spagnuolo, M. Vitelli, Granular control of photovoltaic arrays by means of a multi-output maximum power point tracking algorithm, Progress in Photovoltaics: Research and Applications 21 (5) (2013) 918-932. doi:10.1002/pip. 2179.

URL http://dx.doi .org/10.1002/pip.2179

[21] J. Kivimäki, M. Sitbon, S. Kolesnik, A. Kuperman, T. Suntio, Determining maximum mpptracking sampling frequency for input-voltage-controlled pv-interfacing converter, in: 2016 IEEE Energy Conversion Congress and Exposition (ECCE), 2016, pp. 1-8. doi:10.1109/ ECCE.2016.7855036.

[22] K. Ishaque, Z. Salam, G. Lauss, The performance of perturb and observe and incremental conductance maximum power point tracking method under dynamic weather conditions, Applied Energy 119 (2014) 228-236.

[23] M. Veerachary, T. Senjyu, K. Uezato, Neural-network-based maximum-power-point tracking of coupled-inductor interleaved-boost-converter-supplied PV system using fuzzy controller, IEEE Transactions on Industrial Electronics 50 (4) (2003) 749-758. doi:10.1109/TIE. 2003.814762.

[24] M. Sarvi, S. Ahmadi, S. Abdi, A pso-based maximum power point tracking for photovoltaic systems under environmental and partially shaded conditions, Progress in Photovoltaics: Research and Applications 23 (2) (2015) 201-214. doi:10.1002/pip.2416.

URL http://dx.doi.org/10.1002/pip.2416

[25] F.-S. Pai, R.-M. Chao, S. H. Ko, T.-S. Lee, Performance evaluation of parabolic prediction to maximum power point tracking for PV array, IEEE Transactions on Sustainable Energy 2 (1) (2011) 60-68. doi:10.1109/TSTE.2010.2078844. 
[26] B. Anderson, J. Moore, Optimal Filtering, Prentice-Hall, Englewood Cliffs, NJ, 1979.

[27] S. Motahhir, A. Aoune, A. El Ghzizal, S. Sebti, A. Derouich, Comparison between Kalman filter and incremental conductance algorithm for optimizing photovoltaic energy, Renewables: Wind, Water, and Solar 4 (1) (2017) 8. doi:10.1186/s40807-017-0046-8.

[28] M. Ricco, P. Manganiello, E. Monmasson, G. Petrone, G. Spagnuolo, FPGA-Based Implementation of Dual Kalman Filter for PV MPPT Applications, IEEE Transactions on Industrial Informatics 13 (1) (2017) 176-185. doi:10.1109/TII.2015.2462313.

[29] V. Ramchandani, K. Pamarthi, S. R. Chowdhury, Comparative study of maximum power point tracking using linear kalman filter \& unscented kalman filter for solar photovoltaic array on field programmable gate array, International Journal on Smart Sensing \& Intelligent Systems $5(3)$.

[30] Z. Salam, J. Ahmed, B. S. Merugu, The application of soft computing methods for MPPT of PV system: A technological and status review, Applied Energy 107 (2013) 135-148.

[31] A. Mellit, S. A. Kalogirou, Mppt-based artificial intelligence techniques for photovoltaic systems and its implementation into field programmable gate array chips: Review of current status and future perspectives, Energy 70 (Supplement C) (2014) 1 - 21. doi:https: //doi.org/10.1016/j.energy.2014.03.102

[32] Overall Efficiency of Grid Connected Photovoltaic Inverters, NBN EN 50 530:2010 E, (2010).

[33] R. González-Medina, I. Patrao, G. Garcerá, E. Figueres, A low-cost photovoltaic emulator for static and dynamic evaluation of photovoltaic power converters and facilities, Progress in Photovoltaics: Research and Applications 22 (2) (2014) 227-241.

[34] I. Houssamo, F. Locment, M. Sechilariu, Experimental analysis of impact of MPPT methods on energy efficiency for photovoltaic power systems, International Journal of Electrical Power \& Energy Systems 46 (2013) 98-107.

[35] S. Jain, V. Agarwal, Comparison of the performance of maximum power point tracking schemes applied to single-stage grid-connected photovoltaic systems, Electric Power Applications, IET 1 (5) (2007) 753-762. 
[36] N. Femia, D. Granozio, G. Petrone, G. Spagnuolo, M. Vitelli, Predictive \& adaptive MPPT perturb and observe method, IEEE Transactions on Aerospace and Electronic Systems 43 (3) (2007) 934-950.

[37] A. Hovinen, Fitting of the solar cell iv -curve to the two diode model, Physica Scripta 1994 (T54) (1994) 175.

URL http://stacks . iop.org/1402-4896/1994/i=T54/a=043

[38] S. Suckow, T. M. Pletzer, H. Kurz, Fast and reliable calculation of the twodiode model without simplifications, Progress in Photovoltaics: Research and Applications 22 (4) 494-501. doi: 10.1002/pip.2301.

[39] N. M. A. Alrahim Shannan and N. Z. Yahaya and B. Singh, Single-diode model and two-diode model of pv modules: A comparison, in: IEEE International Conference on Control System, Computing and Engineering, 2013, pp. 210-214. doi:10.1109/ICCSCE.2013.6719960.

[40] N. Mutoh, T. Inoue, A control method to charge series-connected ultraelectric double-layer capacitors suitable for photovoltaic generation systems combining MPPT control method, IEEE Transactions on Industrial Electronics, 54 (1) (2007) 374-383.

[41] S. J. Russell, P. Norvig, Artificial intelligence: A Modern Approach, Pearson Education, 2003.

[42] A. P. Dempster, A generalization of bayesian inference, Journal of the Royal Statistical Society. Series B (Methodological) 30 (2) (1968) 205-247.

[43] C. Paoli, C. Voyant, M. Muselli, M.-L. Nivet, Forecasting of preprocessed daily solar radiation time series using neural networks, Solar Energy 84 (12) (2010) 2146 - 2160. doi:https: //doi.org/10.1016/j.solener.2010.08.011.

[44] M. Flies, N. Charles, Mitigating the Partial Shading Problem by Reswitching of Solar Cells, Master's thesis, KU Leuven, Belgium (2013).

[45] N. Vanparys, Practical Implementation of the Reswitching Algorithm of Solar Cells, Master's thesis, KU Leuven, Belgium (2014).

[46] MATLAB, version 8.3.0.532 (R2014a), The MathWorks Inc., Natick, Massachusetts, 2014. 
[47] S. Liu, R. A. Dougal, Dynamic multi-physics model for solar array, IEEE Power Engineering Review 22 (5) (2002) 66-66.

[48] N. Femia, G. Petrone, G. Spagnuolo, M. Vitelli, Optimizing sampling rate of P\&O MPPT technique, in: 2004 IEEE 35th Annual Power Electronics Specialists Conference (IEEE Cat. No.04CH37551), Vol. 3, 2004, pp. 1945-1949 Vol.3. doi:10.1109/PESC.2004.1355415.

[49] D. P. Hohm, M. E. Ropp, Comparative study of maximum power point tracking algorithms using an experimental, programmable, maximum power point tracking test bed, in: Conference Record of the Twenty-Eighth IEEE Photovoltaic Specialists Conference - 2000 (Cat. No.00CH37036), 2000, pp. 1699-1702. doi:10.1109/PVSC.2000.916230.

[50] L.-R. Chen, C.-H. Tsai, Y.-L. Lin, Y.-S. Lai, A biological swarm chasing algorithm for tracking the PV maximum power point, IEEE Transactions on Energy Conversion 25 (2) (2010) 484493.

[51] B. Bletterie, R. Bruendlinger, S. Spielauer, Quantifying dynamic MPPT performance under realistic conditions first test results - The way forward, in: 21st European Photovoltaic Solar Energy Conference and Exhibition, 2006.

[52] M. Ropp, J. Cale, M. Mills-Price, M. Scharf, S. Hummel, A test protocol to enable comparative evaluation of maximum power point trackers under both static and dynamic irradiance, in: 37th IEEE Photovoltaic Specialists Conference (PVSC), IEEE, 2011, pp. 003734-003737.

[53] B. Lefevre, S. Peeters, J. Poortmans, J. Driesen, Predetermined static configurations of a partially shaded photovoltaic module, Progress in Photovoltaics: Research and Applications 25 (2) (2017) 149-160. 